\title{
Combinatorial invariant theory of projective reflection groups
}

\author{
Fabrizio Caselli \\ Dipartimento di matematica, Università di Bologna, \\ Piazza di Porta San Donato 5, \\ Bologna 40126, Italy
}

\begin{abstract}
We introduce the class of projective reflection groups which includes all complex reflection groups. We show that several aspects involving the combinatorics and the representation theory of complex reflection groups find a natural description in this wider setting.

Résumé. On introduit la classe des groupes de réflexions projectifs, ce qui généralises la notion de groupe engendré par des réflexions. On montre que plusieurs aspects concernants la combinatoire et la théorie des representations des groupes de reflexions complèxes trouvent une description naturelle dans ce cadre plus général.
\end{abstract}

Keywords: Reflection groups, descent statistics, invariant algebras, Young tableaux.

\section{Introduction}

A complex reflection (or simply a reflection) is an endomorphism of a complex vector space $V$ which is of finite order and such that its fixed point space is of codimension 1. Finite reflection groups are finite subgroups of $G L(V)$ generated by reflections. They have probably been introduced by Shephard in (16) and have been characterized by means of their ring of invariants and completely classified by Chevalley (11) and Shephard-Todd (17) in the fifties, generalizing the well-known fundamental theorem of symmetric functions. In this classification there is an infinite family $G(r, p, n)$ of irreducible reflection groups, where $r, p, n$ are positive integers (with $r \equiv 0 \bmod p$ ) and 34 other exceptional groups. The relationship between the combinatorics and the (invariant) representation theory of symmetric groups is fascinating from both combinatorial and algebraic points of view, and the problem of generalizing these sort of results to all reflection groups has been faced in many ways. Besides several results that holds in the full generality of reflection groups, there are some relevant generalizations which have been obtained only for the wreath product groups $G(r, n)=G(r, 1, n)$ (see, e.g., (21, 22; 3, 5; 2)). Some attempts to extend these results to other reflection groups have been made, in particular for Weyl groups of type $D$, (see, e.g., (8; 9: 4) ) though they are probably not completely satisfactory as in the case of wreath products.

In this work we introduce a new class of groups, the projective reflection groups, which are a generalization of reflection groups. We will concentrate our attention on an infinite family $G(r, p, q, n)$ of such groups (where $G(r, p, 1, n)=G(r, p, n)$ in the previous notation). Fundamental in this theory is the 
following notion of duality: if $G=G(r, p, q, n)$ then we denote by $G^{*}=G(r, q, p, n)$ (where the roles of $p$ and $q$ have been interchanged). We note in particular that reflection groups $G$ satisfying $G=G^{*}$ are exactly the wreath products $G(r, n)=G(r, 1,1, n)$ and that in general if $G$ is a reflection group then $G^{*}$ is not. We show that much of the theory of reflection groups can be extended to projective reflection groups and that the combinatorics of a projective reflection group $G=G(r, p, q, n)$ is strictly related to the (invariant) representation theory of $G^{*}$, generalizing several known results for wreath products in a very natural way.

The paper is organized as follows. We present definitions and a characterization in terms of invariants of projective reflection groups in $₫ 2$. We exploit those combinatorial aspects of these groups that we need in $\$ 3$ In $\$ 4$ we further consider the action of a projective reflection group on a ring of polynomials to define and study its coinvariant algebra. In $\$ 5$ we analyze the structure of the irreducible representations of a projective reflection group $G(r, p, q, n)$ and we provide a combinatorial interpretation for their dimensions. In $\$ 6$ we consider a decomposition of the homogeneous components of the coinvariant algebra that leads us to define the descent representations of a projective reflection group: these representations are used in 87 to describe the main new results of this paper. Here we show an explicit basis of the diagonal invariant algebra as a free module over the tensorial invariant algebra of all projective reflection groups $G(r, p, q, n)$. It is in this description that the interplay between a group $G$ and its dual $G^{*}$ attains its apex. In 8 we deduce some properties of the Kronecker coefficients of a projective reflection group that can be deduce from the main results. In $₫ 9$ we extend the Robinson-Schensted correspondence on wreath products to all projective reflection groups of the form $G(r, p, q, n)$ : in this general context it is not a bijection but it will be the key point to solve in $\$ 10$ a problem posed by Barcelo, Reiner and Stanton on the Hilbert series of a certain diagonal invariant module twisted by a Galois automorphism.

\section{Definitions and characterizations}

Let $V$ be a finite dimensional complex vector space and consider the natural map $\varphi: G L(V) \rightarrow$ $G L\left(S^{q}(V)\right)$, where $S^{q}(V)$ is the $q$-th symmetric power of $V$. We clearly have $\operatorname{ker} \varphi=C_{q}$, where $C_{q}$ is the cyclic group of scalar matrices of order $q$ generated by $\zeta_{q} I$, with $\zeta_{q} \stackrel{\text { def }}{=} e^{\frac{2 \pi i}{q}}$.

Now, if $W \subseteq G L(V)$ is a finite reflection group we have $\varphi(W) \cong W /\left(W \cap C_{q}\right)$. In particular, if $C_{q} \subset W$ we have that $W / C_{q}$ can be identified with a subgroup of $G L\left(S^{q}(V)\right)$ by means of the map $\varphi$.

Definition 2.1 Let $G$ be a finite subgroup of $G L\left(S^{q}(V)\right)$. We say that $G$ is a projective reflection group if there exists a reflection group $W \subset G L(V)$ such that $C_{q} \subseteq W$ and $G=W / C_{q}$.

Note that we obtain standard reflection groups in the case $q=1$.

It follows from the previous definition that to classify all possible projective reflection groups we only have to describe all possible scalar subgroups of a reflection group. We know by the work of Shephard and Todd (17) that all but a finite number of irreducible reflection groups are the groups $G(r, p, n)$ that we are going to describe. If $A$ is a matrix with complex entries we denote by $|A|$ the real matrix whose entries are the absolute values of the entries of $A$. The groups $G(r, n)=G(r, 1, n)$ are given by all $n \times n$ matrices satysfying the following conditions:

- the non-zero entries are $r$-th roots of unity;

- there is exactly one non-zero entry in every row and every column (i.e. $|A|$ is a permutation matrix). 
If $p$ divides $r$ then the reflection group $G(r, p, n)$ is the subgroup of $G(r, n)$ given by all matrices $A \in$ $G(r, n)$ such that $\frac{\operatorname{det} A}{\operatorname{det}|A|}$ is a $\frac{r}{p}$-th root of unity.

It is easy to characterize all possible scalar subgroups of the groups $G(r, p, n)$ : in fact we can easily observe that the scalar matrix $\zeta_{q} I$ belongs to $G(r, p, n)$ if and only if $q \mid r$ and $p q \mid r n$.

Definition 2.2 Let $r, p, q, n \in \mathbb{N}$ be such that $p|r, q| r$ and $p q \mid r n$. Then we let

$$
G(r, p, q, n) \stackrel{\text { def }}{=} G(r, p, n) / C_{q}
$$

where $C_{q}$ is the cyclic group generated by $\zeta_{q} I$.

We observe that starting from the wreath product group $G(r, n)$ we could have done first the quotient by the subgroup $C_{q}$ and then taken the subgroup of this quotient consisting of elements $A$ satysfing $\frac{\operatorname{det} A}{\operatorname{det}|A|}$ is a $\frac{r}{p}$-th root of unity (note that this requirement would have been well-defined). We would have obtained the same group $G(r, p, q, n)$ and one of the targets of this paper is to convince the reader that these two operations of "taking subgroups" and "taking quotients" have the same dignity and for many aspects their are dual to each other. In fact, we note the symmetry on the conditions for the parameters $p$ and $q$ in the definition of the group $G(r, p, q, n)$. In particular if $G=G(r, p, q, n)$ then the group $G^{*} \stackrel{\text { def }}{=} G(r, q, p, n)$, where the roles of the parameters $p$ and $q$ are interchanged, is always well-defined. The classical Weyl groups of type $A, B$ and $D$ are respectively in this notation the groups $G(1,1,1, n), G(2,1,1, n)$ and $G(2,2,1, n)$. Note that while Weyl groups of type $A$ and $B$ are fixed by the $*$-operatator, Weyl groups of type $D$ and general reflection groups are not. The main target of this work is to show that the several aspects of the invariant theory of a projective reflection group $G$ is strongly related to and easily described by the combinatorics of $G^{*}$.

One may ask for which choice of the parameters one has $G \cong G^{*}$ as abstract groups.

Proposition 2.3 Let $G=G(r, p, q, n)$, with $n \neq 2$. Then $G \cong G^{*}$ if and only if $\operatorname{GCD}\left(\frac{r n}{p q}, \frac{r}{p}\right)=$ $\operatorname{GCD}\left(\frac{r n}{p q}, \frac{r}{q}\right)$.

Any finite subgroup of $G L(V)$ acts naturally on the symmetric algebra $S\left(V^{*}\right)$. A well-known theorem due to Chevalley and Shephard-Todd says that a finite group $G$ of $G L(V)$ is a reflection group if and only if its invariant ring $S\left(V^{*}\right)^{G}$ is itself a polynomial algebra. Our next target is to generalize this result to the present context. We recall that a projective reflection group is equipped with an action on the symmetric power $S^{q}(V)$. The dual action can be extended to $S_{q}\left[V^{*}\right]$, the algebra of polynomial functions on $V$ generated by homogeneous polynomial functions of degree $q$.

Theorem 2.4 Let $V$ be a complex vector space, $n=\operatorname{dim} V$, and $G$ be a finite group of graded automorphisms of $S_{q}\left[V^{*}\right]$, the algebra generated by homogeneous polynomial functions on $V$ of degree $q$. Then $G$ is a projective reflection group if and only if the invariant algebra $S_{q}\left[V^{*}\right]^{G}$ is generated by $n$ algebraically independent homogeneous elements.

\section{Statistics}

In this section we introduce the main combinatorial tools of projective reflection groups that we need.

If $g \in G(r, n)$ we write $g=\left[\sigma ; c_{1}, \ldots, c_{n}\right]$ if the non-zero entry in the $i$-th row of $g$ is $\zeta_{r}^{c_{i}}$ and $\sigma \in S_{n}$ is the permutation associated to $|g|$ (i.e. $\sigma(i)=j$ if $g_{i, j} \neq 0$ ). We observe that $g$ determines $\sigma$ uniquely 
while the integers $c_{i}$ are determined only modulo $r$. We also note that in this notation we have that $g=\left[\sigma ; c_{1}, \ldots, c_{n}\right]$ belongs to $G(r, p, n)$ if and only if $\sum c_{i} \equiv 0 \bmod p$.

If $g \in G(r, p, q, n)$ we also write $g=\left[\sigma ; c_{1}, \ldots, c_{n}\right]$ to mean that $g$ can be represented by $\left[\sigma ; c_{1}, \ldots, c_{n}\right]$ in $G(r, p, n)$ and we let

$$
\begin{aligned}
\operatorname{HDes}(g) & \stackrel{\text { def }}{=}\left\{i \in[n-1]: c_{i} \equiv c_{i+1} \text { and } \sigma_{i}>\sigma_{i+1}\right\} \\
h_{i}(g) & \stackrel{\text { def }}{=} \#\{j \geq i: j \in \operatorname{HDes}(g)\} \\
k_{i}(g) & \stackrel{\text { def }}{=} \begin{cases}{\left[c_{n}\right]_{r / q}} & \text { if } i=n \\
k_{i+1}+\left[c_{i}-c_{i+1}\right]_{r} & \text { if } i \in[n-1],\end{cases}
\end{aligned}
$$

where $[c]_{s}$ is the smallest non negative representative of the class of the integer $c$ modulo $s$.

Note that these statistics do not depend on the choice of the integers $c_{1}, \ldots, c_{n}$ for representing $g$. For example, let $g=[27648153 ; 2,3,3,5,1,7,3,2] \in G(6,2,3,8)$. Then $\operatorname{HDes}(g)=\{2,5\},\left(h_{1}, \ldots, h_{8}\right)=$ $(2,2,1,1,1,0,0,0)$ and $\left(k_{1}, \ldots, k_{8}\right)=(18,13,13,9,5,5,1,0)$.

If $q=p=1$ these statistics give an alternative definition for the flag-major index of Adin and Roichman (see (3)) for wreath products $G(r, n)$. In fact, if we let

$$
\operatorname{Des}(g)=\left\{i: \text { either }\left[c_{i}\right]_{r}<\left[c_{i+1}\right]_{r} \text { or }\left[c_{i}\right]_{r}=\left[c_{i+1}\right]_{r} \text { and } \sigma_{i}>\sigma_{i+1}\right\},
$$

then the flag-major index is defined as

$$
\operatorname{fmaj}(g) \stackrel{\text { def }}{=} r \sum_{i \in \operatorname{Des}(g)} i+\sum_{i}\left[c_{i}\right]_{r}
$$

and we can easily verify that in this case we have fmaj $(g)=\sum\left(r \cdot h_{i}(g)+k_{i}(g)\right)$.

We note that if $\lambda_{i}(g) \stackrel{\text { def }}{=} r \cdot h_{i}(g)+k_{i}(g)$ then the sequence $\lambda(g) \stackrel{\text { def }}{=}\left(\lambda_{1}(g), \ldots, \lambda_{n}(g)\right)$ is a partition. We may also observe that $\lambda(g)$ is such that $g=[|g| ; \lambda(g)]$ and that $\lambda(g)$ is the minimal partition (with respect to containment of the corresponding Ferrers diagram) satysfing this condition.

Extending the notion of flag-major index we define the flag-major index for the projective reflection group $G(r, p, q, n)$ by fmaj $(g) \stackrel{\text { def }}{=}|\lambda(g)|$.

\section{The coinvariant algebra}

As we observed in $\$ 2$ we have an action of any projective reflection group $G$ on the algebra $S_{q}\left[V^{*}\right]$ generated by homogeneous polynomial functions on $V$ of degree $q$. We denote by $I_{+}^{G}$ the ideal of $S_{q}\left[V^{*}\right]$ generated by homogeneous elements in $S_{q}\left[V^{*}\right]^{G}$ of positive degree, and define the coinvariant algebra of $G$ by

$$
R^{G} \stackrel{\text { def }}{=} S_{q}\left[V^{*}\right] / I_{+}^{G}
$$

The coinvariant algebra $R^{G}$ is a graded representation of $G$ and, as it was the case for reflection groups, it is isomorphic as a $G$-module to the group algebra $\mathbb{C} G$.

Proposition 4.1 If $G$ is any projective reflection group, we have an isomorphism of $G$-modules $R^{G} \cong$ $\mathbb{C} G$. 
If the projective reflection group $G$ is of the form $G=G(r, p, q, n)$ we can describe the coinvariant algebra in a more explicit way. It could be natural to expect a basis for the algebra $R^{G}$ indexed by elements of $G$. As it was mentioned in the introduction, this is the first occurrence of the invariant theory of a projective reflection group $G$ which is naturally described by its dual group $G^{*}$. Generalizing and unifying results and definitions in $(1 ; 8 ; 4)$, we associate to any element $g \in G$ a monomial $a_{g} \in \mathbb{C}[X] \stackrel{\text { def }}{=}$ $\mathbb{C}\left[x_{1}, \ldots, x_{n}\right]$ of degree $\operatorname{fmaj}(g)$ in the following way

$$
a_{g}(X) \stackrel{\text { def }}{=} \prod_{i} x_{|g|(i)}^{\lambda_{i}(g)}
$$

We denote by $S_{q}[X]$ the algebra of polynomials in $\mathbb{C}[X]$ generated by the monomials of degree $q$. Then it is not difficult to verify that

$$
a_{g} \in S_{p}[X]
$$

for all $g \in G(r, p, q, n)$.

Theorem 4.2 If $G=G(r, p, q, n)$ then the set $\left\{a_{g}: g \in G^{*}\right\}$ represents a basis for $R^{G}$.

\section{The irreducible representations}

In this section we describe explicitly a natural parametrization of the irreducible representations of a projective reflection group $G(r, p, q, n)$. Given a partition $\mu$ of $n$, the Ferrers diagram of shape $\mu$ is a collection of boxes, arranged in left-justified rows, with $\mu_{i}$ boxes in row $i$. We denote by $\operatorname{Fer}(r, p, n)$ the set of $r$-tuples of Ferrers diagrams whose shapes $\left(\lambda^{(0)}, \ldots, \lambda^{(r-1)}\right)$ are such that $\sum\left|\lambda^{(i)}\right|=n$ and $\sum_{i} i\left|\lambda^{(i)}\right| \equiv 0 \bmod p$. This may recall the definition of $G(r, p, n)$ where the role of $\sum_{i} c_{i}(g)$ is played by $\sum_{i} i\left|\lambda^{(i)}\right|$. In an extreme parallelism with the groups $G(r, p, n)$ we have the following result.

Lemma 5.1 Let $\left(\lambda^{(0)}, \ldots, \lambda^{(r-1)}\right) \in \operatorname{Fer}(r, p, n)($ and $q \in \mathbb{N}$ be such that $q \mid r$ and $p q \mid r n)$. Then

$$
\left(\lambda^{(r / q)}, \ldots, \lambda^{(r-1+r / q)}\right) \in \operatorname{Fer}(r, p, n)
$$

where $\lambda^{(j)} \stackrel{\text { def }}{=} \lambda^{(j-r)}$ if $j \geq r$.

If $\mu \in \operatorname{Fer}(r, p, n)$ we denote by $\mathcal{S} \mathcal{T}_{\mu}$ the set of all possible fillings of the boxes in $\mu$ with all the numbers from 1 to $n$ appearing once, in such way that rows are increasing from left to right and columns are incresing from top to bottom in every single Ferrers diagram of $\mu$. Moreover we let $\mathcal{S T}(r, p, n) \stackrel{\text { def }}{=}$ $\cup_{\mu \in \operatorname{Fer}(r, p, n)} \mathcal{S T}_{\mu}$.

By Lemma 5.1 we have a natural action of $C_{q}$ on both $\operatorname{Fer}(r, p, n)$ and $\mathcal{S} \mathcal{T}(r, p, n)$. We denote the corresponding quotient sets by $\operatorname{Fer}(r, p, q, n)$ and $\mathcal{S T}(r, p, q, n)$. If $T \in \mathcal{S T}(r, p, q, n)$ we denote by $\mu(T)$ its corresponding shape in $\operatorname{Fer}(r, p, q, n)$ and if $\mu \in \operatorname{Fer}(r, p, q, n)$ we let $\mathcal{S} \mathcal{T}_{\mu} \stackrel{\text { def }}{=}\{T \in \mathcal{S T}(r, p, q, n)$ : $\mu(T)=\mu\}$. Finally, if Fer $=\operatorname{Fer}(r, p, q, n)$, we let Fer $\stackrel{\text { def }}{=} \operatorname{Fer}(r, q, p, n)$.

Proposition 5.2 The irreducible representations of $G(r, p, q, n)$ are naturally parametrized by pairs $(\mu, \rho)$, where $\mu \in$ Fer* $^{*}$ and $\rho \in\left(C_{p}\right)_{\mu}$, the stabilizer of any element in the class $\mu$. Moreover the dimension of the irreducible representation indexed by $(\mu, \rho)$ is independent of $\rho$ and it is equal to $\left|\mathcal{S} \mathcal{T}_{\mu}\right|$.

If $\phi$ is an irreducible representation of $G$ indexed by a pair $(\mu, \rho)$ we let $\mu(\phi) \stackrel{\text { def }}{=} \mu \in$ Fer* $^{*}$. 


\section{The descent representations}

If $M$ is a monomial in $\mathbb{C}[X]$ we denote by $\lambda(M)$ its exponent partition, i.e. the partition obtained by rearranging the exponents of $M$. We say that a polynomial is homogeneous of partition degree $\lambda$ if it is a linear combination of monomials whose exponent partition is $\lambda$. If $G=G(r, p, q, n)$ and $|\lambda| \equiv 0 \bmod q$, we can consider the submodule $R_{\unlhd \lambda}^{G}$ of $R^{G}$ spanned by monomials of total degree $|\lambda|$ and partition degree $\unlhd \lambda$ and we can similarly define $R_{\triangleleft \lambda}^{G}$. Here $\unlhd$ and $\triangleleft$ mean smaller and strictly smaller in the dominance order of partitions. Following and generalizing $(1,8,4)$ we denote the quotient module by

$$
R_{\lambda}^{G \stackrel{\text { def }}{=}} R_{\unlhd \lambda}^{G} / R_{\triangleleft \lambda}^{G}
$$

We call the modules $R_{\lambda}^{G}$ the descent representations of $G$. A straightforward application of Maschke's theorem implies that, for any $k \equiv 0 \bmod q$, we have an isomorphism

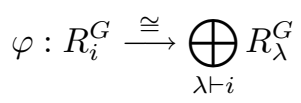

such that every element in $\varphi^{-1}\left(R_{\lambda}^{G}\right)$ can be represented by a homogeneous polynomial in $S_{q}[X]$ of partition degree $\lambda$. We recall that if $g \in G^{*}$ then the monomial $a_{g}$ has partition degree $\lambda(g)$ and so it represents an element in $R_{\lambda(g)}^{G}$.

Lemma 6.1 Let $\lambda$ be a partition such that $|\lambda| \equiv 0 \bmod q$. Then the set

$$
\left\{a_{g}: g \in G^{*} \text { and } \lambda(g)=\lambda\right\}
$$

is a system of representatives of a basis of $R_{\lambda}^{G}$. In particular $\operatorname{dim}\left(R_{\lambda}^{G}\right)=\left|\left\{g \in G^{*}: \lambda(g)=\lambda\right\}\right|$.

Our next target is an explicit description of the irreducible decomposition of the modules $R_{\lambda}^{G}$. We can define the statistics $h_{i}$ and $k_{i}$ in $\mathcal{S T}(r, p, q, n)$ similarly to the case of $G(r, p, q, n)$. Let $T \in \mathcal{S T}(r, p, q, n)$ be represented by $\left(T_{1}, \ldots, T_{r}\right)$. For $i \in[n]$ we let $c_{i}=j$ if $i \in T_{j}$.

$$
\begin{aligned}
\operatorname{HDes}(T) & \stackrel{\text { def }}{=}\left\{i \in[n-1]: c_{i}=c_{i+1} \text { and } i \text { appears strictly above } i+1\right\} \\
h_{i}(T) & \stackrel{\text { def }}{=} \#\{j \geq i: j \in \operatorname{HDes}(T)\} \\
k_{i}(T) & \stackrel{\text { def }}{=} \begin{cases}{\left[c_{n}\right]_{r / q}} & \text { if } i=n \\
k_{i+1}+\left[c_{i}-c_{i+1}\right]_{r} & \text { if } i \in[n-1]\end{cases}
\end{aligned}
$$

It is clear that these definitions do not depend on the choice of the representative $\left(T_{1}, \ldots, T_{r}\right)$. For example if

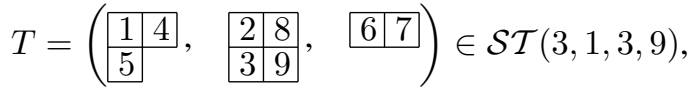

we have $\left(h_{1}, \ldots, h_{9}\right)=(3,3,2,2,1,1,1,1,0)$ and $\left(k_{1}, \ldots, k_{9}\right)=(5,3,3,2,2,1,1,0,0)$. We define $\lambda_{i}(T) \stackrel{\text { def }}{=} r h_{i}(T)+k_{i}(T), \lambda(T)=\left(\lambda_{1}(T), \ldots, \lambda_{n}(T)\right.$ and fmaj $(T)=|\lambda(T)|$.

Proposition 6.2 Let $\phi$ be an irreducible representation of $G$. Then the multiplicity of $\phi$ in $R_{\lambda}^{G}$ is given by

$$
\left\langle\chi^{\phi}, \chi^{R_{\lambda}^{G}}\right\rangle=\mid\left\{T \in \mathcal{S} \mathcal{T}_{\mu(\phi)}: \lambda(T)=\lambda \mid,\right.
$$

where $\mu(\phi) \in$ Fer* $^{*}$ is defined at the end of $\$ 5$. 
This proposition unifies and generalizes the corresponding coarse results of Lusztig (unpublished) and Kraśkiewicz-Weyman (14) in Type A and Stembridge (22) for reflection groups and the corresponding refined results of Adin-Brenti-Roichman (1) in Type A and B and of Bagno-Biagioli (4) for reflection groups.

\section{Tensorial and diagonal actions}

In this section we describe the main result of this work (Theorem 7.5) which present an explicit basis for the diagonal invariant algebra of a projective reflection group $G=G(r, p, q, n)$ (considered as a free module over the tensorial invariant algebra) in terms of the dual group $G^{*}$. This result is new also in the generality of reflection groups (see (7), 6) for related results in type $A$ and $B$ ). Here it is really apparent that not only the combinatorics of $G^{*}$ (as in the previous sections) but also its algebraic structure play a crucial role in the invariant theory of $G$. Let $S_{q}[X]^{\otimes k}$ be the $k$-th tensor power of the algebra of polynomials $S_{q}[X]$ defined in $\$ 4$. On this algebra we consider the natural action of the group $G^{k}$ (where the $i$-th coordinate of $G^{k}$ acts on the $i$-th factor in $S_{q}[X]^{\otimes k}$ ) and of its diagonal subgroup $\Delta G$. We are particularly interested in the corresponding invariant algebras. Every monomial in $S_{q}[X]^{\otimes k}$ can be described by a $k \times n$-matrix with non negative integer entries such that the sum in each row is divided by $q$. To any such matrix $A$ we associate the monomial $\mathcal{X}^{A} \stackrel{\text { def }}{=} \prod_{i, j} x_{i, j}^{a_{i, j}}$. Here and in what follows we identify $S_{q}[X]^{\otimes k}$ with the algebra of polynomials $S_{q}\left[X_{1}, \ldots, X_{k}\right]=S_{q}\left[x_{i, j}\right]$ (where $i \in[k]$ and $j \in[n]$ ) spanned by monomials whose degree in $x_{i, 1}, \ldots, x_{i, n}$ is a multiple of $q$ for all $i \in[k]$. We refer to the algebra $S_{q}\left[X_{1}, \ldots, X_{k}\right]^{\Delta G}$ as the diagonal invariant algebra of $G$. It is clear that $S_{q}\left[X_{1}, \ldots, X_{k}\right]^{\Delta G}$ is generated by the polynomials

$$
\left(\mathcal{X}^{A}\right) \stackrel{\text { def }}{=} \frac{1}{|G|} \sum_{g \in \Delta G} g\left(\mathcal{X}^{A}\right) .
$$

Lemma 7.1 Let $A$ be a $k \times n$ matrix with row sums divided by $q$ and let $s_{i}$ be the sum of the entries in its $i$-th column. Then $\left(\mathcal{X}^{A}\right)^{\#} \neq 0$ if and only if the following two conditions are satisfied

1. $s_{i} \equiv s_{j}$ for all $i, j$;

2. $p s_{i} \equiv 0$ for all $i$.

We recall that a $k$-partite partition (see $(12,13)$ ) is a $k \times n$ matrix $A=\left(a_{i, j}\right)$ with non-negative integer entries such that $a_{i, j} \geq a_{i, j+1}$ whenever $a_{h, j}=a_{h, j+1}$ for all $h<i$. We denote by $\mathcal{B}_{k}(r, p, q, n)$ the set of $k \times n$-matrices which are $k$-partite partitions, with row sums divided by $q$ and column sums satisfying (1) and (2) in Lemma 7.1.

Corollary 7.2 The set $\left\{\left(\mathcal{X}^{A}\right)^{\#}: A \in \mathcal{B}_{k}(r, p, q, n)\right\}$ is a basis for the diagonal invariant algebra of $G$.

We recall that the algebra $S_{q}\left[X_{1}, \ldots, X_{k}\right]^{\Delta G}$, being Cohen-Macaulay (see (19, Proposition 3.1)), is a free module over its subalgebra $S_{q}\left[X_{1}, \ldots, X_{k}\right]^{G^{k}}$ and our next target is the description of a basis for $S_{q}\left[X_{1}, \ldots, X_{k}\right]^{\Delta G}$ as a free $S_{q}\left[X_{1}, \ldots, X_{k}\right]^{G^{k}}$-module.

Definition 7.3 Let $\lambda$ be a partition with $n$ parts and $g \in G(r, p, q, n)$. We say that $\lambda$ is $g$-compatible if $\lambda-\lambda(g)$ is a partition and $g=[|g| ; \lambda]$. 
We note that in the case of the symmetric group the condition $g=[|g| ; \lambda]$ in the previous definition is empty and we obtain an equivalent definition of a $\sigma$-compatible partition given in (12). The special case of the following result where $G$ is the symmetric group is proved in (12).

Theorem 7.4 There is a bijection between $\mathcal{B}_{k}(r, p, q, n)$ and $(2 k)$-tuples $\left(g_{1}, \ldots, g_{k} ; \lambda^{(1)}, \ldots, \lambda^{(k)}\right)$ where

- $g_{1}, \ldots, g_{k} \in G^{*}$ are such that $g_{1} \cdots g_{k}=1$;

- $\lambda^{(i)}$ is a $g_{i}$-compatible partition.

The bijection is given by

$$
\Phi\left(g_{1}, \ldots, g_{k} ; \lambda^{(1)}, \ldots, \lambda^{(k)}\right)=\left(\begin{array}{llll}
\lambda_{1}^{(1)} & \lambda_{2}^{(1)} & \cdots & \lambda_{n}^{(1)} \\
\lambda_{\sigma_{1}(1)}^{(2)} & \lambda_{\sigma_{1}(2)}^{(2)} & \cdots & \lambda_{\sigma_{1}(n)}^{(2)} \\
\lambda_{\left(\sigma_{1} \ldots \sigma_{k-1}\right)(1)}^{(k)} & \lambda_{\left(\sigma_{1} \ldots \sigma_{k-1}\right)(2)}^{(k)} & \cdots & \lambda_{\left(\sigma_{1} \ldots \sigma_{k-1}\right)(n)}^{(k)}
\end{array}\right),
$$

where $\sigma_{i}=\left|g_{i}\right|$ and the composition of permutations is from left to right.

If $g_{1}, \ldots, g_{k} \in G^{*}$ and $g_{1} \cdots g_{k}=1$ we let

$$
A\left(g_{1}, \ldots, g_{k}\right) \stackrel{\text { def }}{=} \Phi\left(g_{1}, \ldots, g_{k} ; \lambda\left(g_{1}\right), \ldots, \lambda\left(g_{k}\right)\right) .
$$

With this terminology Theorem 7.4 can be restated as follows: if $A \in \mathcal{B}_{k}(r, p, q, n)$ then there exist unique $g_{1}, \ldots, g_{k} \in G^{*}$ with $g_{1} \cdots g_{k}=1$ such that

$$
\mathcal{X}^{A}=\mathcal{X}^{A\left(g_{1}, \ldots g_{k}\right)} M_{1}\left(X_{1}\right) \cdots M_{k}\left(X_{k}\right),
$$

where, for all $i \in[k], M_{i}$ is a monomial such that $\lambda\left(M_{i}\right)=\lambda_{i}\left(\mathcal{X}^{A}\right)-\lambda\left(g_{i}\right)$ is a partition whose parts are all congruent to the same multiple of $r / p$ modulo $r$. Here $\lambda_{i}\left(\mathcal{X}^{A}\right)$ is the exponent partition of $\mathcal{X}^{A}$ with respect to the variables $x_{i, 1}, \ldots, x_{i, n}$. This is the main point in the proof of the following result.

Theorem 7.5 The set of polynomials

$$
\left\{\left(\mathcal{X}^{A\left(g_{1}, \ldots, g_{k}\right)}\right)^{\#}: g_{1}, \ldots, g_{k} \in G^{*} \text { and } g_{1} \cdots g_{k}=1\right\}
$$

is a basis for $S_{q}\left[X_{1}, \ldots, X_{k}\right]^{\Delta G}$ as a free module over $S_{q}\left[X_{1}, \ldots, X_{k}\right]^{G^{k}}$.

An immediate consequence of Theorem 7.5 is the following equality

$$
\frac{\operatorname{Hilb}\left(S_{q}\left[X_{1}, \ldots, X_{k}\right]^{\Delta G}\right)\left(y_{1}, \ldots, y_{k}\right)}{\operatorname{Hilb}\left(S_{q}\left[X_{1}, \ldots, X_{k}\right]^{G^{k}}\right)\left(y_{1}, \ldots, y_{k}\right)}=\sum_{\substack{g_{1}, \ldots, g_{k} \in G^{*} \\ g_{1} \cdots g_{k}=1}} y_{1}^{\mathrm{fmaj}\left(g_{1}\right)} \cdots y_{k}^{\mathrm{fmaj}\left(g_{k}\right)} .
$$

Theorem 7.5 and its proof allow us to obtain an important refinement of the previous identity. The algebra $S_{q}\left[X_{1}, \ldots, X_{k}\right]$ is multigraded by $k$-tuples of partitions with at most $n$ parts: we just say that a monomial $M$ is homogeneous of multipartition degree $\left(\lambda^{(1)}, \ldots, \lambda^{(k)}\right)$ if its exponent partition with respect to the variables $x_{i, 1}, \ldots x_{i, n}$ is $\lambda^{(i)}$ for all $i$. If we consider the Hilbert series of the invariant algebras above with respect to this multipartition degree we obtain the following result. 
Corollary 7.6 We have

$$
\frac{\operatorname{Hilb}\left(S_{q}\left[X_{1}, \ldots, X_{k}\right]^{\Delta G}\right)\left(Y_{1}, \ldots, Y_{k}\right)}{\operatorname{Hilb}\left(S_{q}\left[X_{1}, \ldots, X_{k}\right]^{G^{k}}\right)\left(Y_{1}, \ldots, Y_{k}\right)}=\sum_{\substack{g_{1}, \ldots, g_{k} \in G^{*}: \\ g_{1} \cdots g_{k}=1}} Y_{1}^{\lambda\left(g_{1}\right)} \cdots Y_{k}^{\lambda\left(g_{k}\right)},
$$

where $Y_{i}=\left(y_{i, 1}, \ldots, y_{i, n}\right)$.

\section{The Kronecker coefficients}

We can use the descent representations of a projective reflection group introduced in 86 to give to the coinvariant algebra the structure of a partition-graded module. By means of this grading of the coinvariant algebra we can also decompose the algebra

$$
\frac{S_{q}\left[X_{1}, \ldots, X_{k}\right]}{I_{+}^{G^{k}}} \cong \underbrace{R^{G} \otimes \cdots \otimes R^{G}}_{k}
$$

and its diagonal invariant subalgebra

$$
\left(\frac{S_{q}\left[X_{1}, \ldots, X_{k}\right]}{I_{+}^{G^{k}}}\right)^{\Delta G} \cong \frac{S_{q}\left[X_{1}, \ldots, X_{k}\right]^{\Delta G}}{J_{+}^{G^{k}}}
$$

in homogeneous components whose degrees are $k$-tuples of partitions with at most $n$ parts. Here $I_{+}^{G^{k}}$ and $J_{+}^{G^{k}}$ are the ideals generated by homogeneous $G^{k}$-invariant polynomials of positive degree inside $S_{q}\left[X_{1}, \ldots, X_{k}\right]$ and $S_{q}\left[X_{1}, \ldots, X_{k}\right]^{\Delta G}$ respectively.

We define the refined fake degree polynomial $f^{\phi}\left(y_{1}, \ldots, y_{n}\right)$ of a projective reflection group $G$ as the polynomial whose coefficient of $y_{1}^{\lambda_{1}} \cdots y_{n}^{\lambda_{n}}$ is the multiplicity of the irreducible representation $\phi$ of $G$ in $R_{\lambda}^{G}$. If $\phi_{1}, \ldots, \phi_{k}$ are $k$ irreducible representations of $G$ we define the Kronecker coefficients of $G$ by

$$
g_{\phi_{1}, \ldots, \phi_{k}} \stackrel{\text { def }}{=} \frac{1}{|G|} \sum_{g \in G} \chi^{\phi_{1}}(g) \cdots \chi^{\phi_{k}}(g)
$$

If $G=G(r, p, q, n)$ and $\mu_{1}, \ldots, \mu_{k} \in \operatorname{Fer}^{*}=\operatorname{Fer}(r, q, p, n)$, we define the coarse Kronecker coefficients of $G$ by

$$
g_{\mu_{1}, \ldots, \mu_{k}} \stackrel{\text { def }}{=} \sum_{i} \sum_{\phi_{i}: \mu\left(\phi_{i}\right)=\mu_{i}} g_{\phi_{1}, \ldots, \phi_{k}} .
$$

The following result is a consequence of (18, Theorem 5.11).

Theorem 8.1 We have

$$
\operatorname{Hilb}\left(\frac{S_{q}\left[X_{1}, \ldots, X_{k}\right]^{\Delta G}}{J_{+}^{G^{k}}}\right)\left(Y_{1}, \ldots, Y_{k}\right)=\sum_{\phi_{1}, \ldots, \phi_{k} \in \operatorname{Irr}(G)} g_{\phi_{1}, \ldots, \phi_{k}} f^{\phi_{1}}\left(Y_{1}\right) \cdots f^{\phi_{k}}\left(Y_{k}\right)
$$

where the sum is taken over all k-tuples of irreducible representations of $G$. 
By Proposition 6.2 we deduce that Theorem 8.1 can be restated as follows

$$
\operatorname{Hilb}\left(\frac{S_{q}\left[X_{1}, \ldots, X_{k}\right]^{\Delta G}}{J_{+}^{G^{k}}}\right)\left(Y_{1}, \ldots, Y_{k}\right)=\sum_{T_{1}, \ldots, T_{k} \in \mathcal{S} \mathcal{T}^{*}} g_{\mu\left(T_{1}\right), \ldots, \mu\left(T_{k}\right)} Y_{1}^{\lambda\left(T_{1}\right)} \cdots Y_{k}^{\lambda\left(T_{k}\right)},
$$

where $\mathcal{S} \mathcal{T}^{*} \stackrel{\text { def }}{=} \mathcal{S} \mathcal{T}(r, q, p, n)$. So, by Theorem 7.5 and Corollary 7.6 we have the following result.

Corollary 8.2 Let $G=G(r, p, q, n)$ and $\mathcal{S} \mathcal{T}=\mathcal{S} \mathcal{T}(r, p, q, n)$. Then

$$
\sum_{\substack{g_{1}, \ldots, g_{k} \in G \\ g_{1} \cdots g_{k}=1}} Y_{1}^{\lambda\left(g_{1}\right)} \cdots Y_{k}^{\lambda\left(g_{k}\right)}=\sum_{T_{1}, \ldots, T_{k} \in \mathcal{S} \mathcal{T}} g_{\mu\left(T_{1}\right), \ldots, \mu\left(T_{k}\right)} Y_{1}^{\lambda\left(T_{1}\right)} \cdots Y_{k}^{\lambda\left(T_{k}\right)} .
$$

We observe that Corollary 8.2 provides us a purely combinatorial algorithm to compute the coarse Kronecker coefficients of $G$. This can be achieved in a way which is similar to the corresponding result for the symmetric group (see $(10, \S 4)$ ).

In the next section we describe a bijective proof of Corollary 8.2 in the case $k=2$.

\section{The Robinson-Schensted correspondence}

Recall the classical Robinson-Schensted correspondence from (20, §7.11)). This correspondence has been extended to wreath product groups $G(r, n)$ in (21) in the following way. Given $g \in G(r, n)$ and $j \in[0, r-1]$, we let $\left\{i_{1}, \ldots, i_{h}\right\}=\left\{l \in[n]: c_{l}(g)=j\right\}$ and we consider the two-line array $A_{j}=$ $\left(\begin{array}{cccc}i_{1} & i_{2} & \cdots & i_{h} \\ \sigma\left(i_{1}\right) & \sigma\left(i_{2}\right) & \cdots & \sigma\left(i_{h}\right)\end{array}\right)$, where $\sigma=|g|$, and the pair of tableaux $\left(P_{j}, Q_{j}\right)$ obtained by applying the Robinson-Schensted correspondence to $A_{j}$. Then the Stanton-White correspondence

$$
g \mapsto(P(g), Q(g)) \stackrel{\text { def }}{=}\left(\left(P_{0}, \ldots, P_{r-1}\right),\left(Q_{0}, \ldots, Q_{r-1}\right)\right)
$$

is a bijection between $G(r, n)$ and pairs of tableaux of the same shape in $\mathcal{S T}(r, 1, n)$. Furthermore we have $\lambda(g)=\lambda(Q(g))$ and $\lambda\left(\bar{g}^{-1}\right)=\lambda(P(g))$.

Now let $g \in G(r, p, q, n)$ and $\tilde{g} \in G(r, p, n)$ be a lifting of $g$. Then the classes in $\mathcal{S} \mathcal{T}(r, p, q, n)$ of the tableaux $P(\tilde{g})$ and $Q(\tilde{g})$ obtained by applying the previous correspondence depend uniquely on $g$ and not on the lifting $\tilde{g}$. Therefore one can define a map $g \mapsto(P(g), Q(g))$ which associates to any element in $G(r, p, q, n)$ a pair of tableaux in $\mathcal{S T}(r, p, q, n)$ of the same shape. The following result is the natural generalization of the Stanton-White correspondence to projective reflection groups.

Theorem 9.1 Let $P, Q$ be two tableaux in $\mathcal{S} \mathcal{T}(r, p, q, n)$ of the same shape $\mu$. Then

$$
\mid\{g \in G(r, p, q, n): P(g)=P \text { and } Q(g)=Q\}|=|\left(C_{q}\right)_{\mu} \mid,
$$

where $\left(C_{q}\right)_{\mu}$ is the stabilizer in $C_{q}$ of any element in the class $\mu$.

We observe that Theorem 9.1 provides a bijective proof that

$$
|G|=\sum_{\phi \in \operatorname{Irr}\left(G^{*}\right)}(\operatorname{dim} \phi)^{2},
$$

since $\operatorname{dim} \phi=\left|\mathcal{S} \mathcal{T}_{\mu(\phi)}\right|$ and, given $\mu \in$ Fer, we have $\left|\left\{\phi \in \operatorname{Irr}\left(G^{*}\right): \mu(\phi)=\mu\right\}\right|=\left|\left(C_{q}\right)_{\mu}\right|$. 


\section{Galois automorphisms}

The next target is to use the theory developed in the previous sections to solve a problem posed in (5, Question 6.3). The objects of our study here are again Hilbert series of invariant algebras as in \$7 but with a new ingredient given by a Galois automorphism. Given any projective reflection group $G$ (not necessarily of the form $G(r, p, q, n)$ ) we consider a cyclotomic field $\mathbb{Q}\left[e^{\frac{2 \pi i}{m}}\right]$ which contains the entries of the (representatives of the) elements in $G$. Then we observe that for any $\sigma \in \operatorname{Gal}\left(\mathbb{Q}\left[e^{\frac{2 \pi i}{m}}\right], \mathbb{Q}\right)$ we have $\sigma\left(C_{q}\right)=C_{q}$ and so we can consider the group $G^{\sigma} \stackrel{\text { def }}{=} \sigma(G)$ obtained by applying $\sigma$ to the entries of the representatives of the elements of $G$. We observe that if $G=G(r, p, q, n)$ then, since $\sigma\left(\zeta_{r}\right)=\zeta_{r}^{d}$ for some $d$ such that $G C D(r, d)=1$, we have that $G^{\sigma}=G$, i.e. $\sigma \in \operatorname{Aut}(G)$. The setting is similar to that of 87 with $k=2$ : we consider the following twisted diagonal subgroup of $G \times G^{\sigma}$

$$
\Delta^{\sigma} G \stackrel{\text { def }}{=}\left\{\left(g, g^{\sigma}\right): g \in G\right\}
$$

where $g^{\sigma} \stackrel{\text { def }}{=} \sigma(g)$. We recall that $G \times G^{\sigma}$ acts on the symmetric algebra $S_{q}\left[X_{1}, X_{2}\right]$ and that this algebra has a bipartition degree given by the exponent partitions in the two sets of variables. The coinvariant algebra of $R^{G \times G^{\sigma}}$ is canonically isomorphic to $R^{G} \otimes R^{G^{\sigma}}$ and so it also affords a bipartition degree given by

$$
R_{\lambda^{(1)}, \lambda^{(2)}}^{G \times G^{\sigma}} \cong R_{\lambda^{(1)}}^{G} \otimes R_{\lambda^{(2)}}^{G^{\sigma}}
$$

We are interested in the subalgebra of $R^{G \times G^{\sigma}}$ consisting of $\Delta^{\sigma} G$-invariants and in particular to its Hilbert series with respect to the bipartition degree defined above.

The following result was proved in (5) for reflection groups in its unrefined version (i.e. considering only the bidegree in $\mathbb{N}^{2}$ and not the bipartition degree).

Theorem 10.1 Let $G$ be any projective reflection group. Then

$$
G^{\sigma}\left(Y_{1}, Y_{2}\right) \stackrel{\text { def }}{=} \operatorname{Hilb}\left(\frac{S_{q}\left[X_{1}, X_{2}\right]^{\Delta^{\sigma} G}}{J_{+}^{G \times G^{\sigma}}}\right)\left(Y_{1}, Y_{2}\right)=\sum_{\phi \in \operatorname{Irr}(G)} f^{\sigma \phi}\left(Y_{1}\right) f^{\bar{\phi}}\left(Y_{2}\right) .
$$

If $G$ is of the form $G=G(r, p, q, n)$, by Theorem 9.1, then the polynomial $G^{\sigma}\left(Y_{1}, Y_{2}\right)$ takes the following simple form in terms of the dual group $G^{*}$.

Corollary 10.2 For any projective reflection group $G=G(r, p, q, n)$ and any Galois automorphism $\sigma \in \operatorname{Gal}\left(\mathbb{Q}\left[e^{\frac{2 \pi i}{m}}\right] / \mathbb{Q}\right)$ we have

$$
G^{\sigma}\left(Y_{1}, Y_{2}\right)=\sum_{g \in G^{*}} Y_{1}^{\lambda\left(g^{\sigma}\right)} Y_{2}^{\lambda\left(g^{-1}\right)} .
$$

The unrefined version of the previous corollary

$$
G^{\sigma}\left(y_{1}, y_{2}\right)=\sum_{g \in G^{*}} y_{1}^{\mathrm{fmaj}\left(g^{\sigma}\right)} y_{2}^{\operatorname{fmaj}\left(g^{-1}\right)}
$$

provides an answer to (5, Question 6.3). We believe that one can generalize these facts to a multivariate setting using Corollaries 7.6 and 8.2 instead of Theorem 9.1 . 


\section{References}

[1] R. Adin, F. Brenti and Y. Roichman, Descent representations and multivariate statistics, Trans. Amer. Math. Soc. 357 (2005), no. 8, 3051-3082

[2] R. Adin, A. Postnikov and Y. Roichman, A Gelfand model for wreath products, preprint.

[3] R. Adin and Y. Roichman, The flag major index and group actions on polynomial rings, Europ. J. Combin. 22 (2001), 431-446.

[4] E. Bagno and R. Biagioli, Colored-descent representations of complex reflection groups $G(r, p, n)$. Israel J. Math. 160 (2007), 317-347

[5] H. Barcelo, V. Reiner and D. Stanton, Bimahonian distributions, J. London Math. Soc. 77 (2008), 627-646.

[6] F. Bergeron and R. Biagioli, Tensorial square of the hyperoctahedral group coinvariant space, Electron. J. Combin., 13 (2006), R38.

[7] F. Bergeron and F. Lamontagne, Decomposition of the diagonal action of $S_{n}$ on the coinvariant space of $S_{n} \times S_{n}$, Sém. Lothar. Combin. 52 (2004/07), Art. B52e.

[8] R. Biagioli and F. Caselli, Invariant algebras and major indices for classical Weyl groups, Proc. London Math. Soc. (3) 88 (2004), no. 3, 603-631.

[9] R. Biagioli and F. Caselli, A descent basis for the coinvariant algebra of type D. J. Algebra 275 (2004), no. 2, 517-539.

[10] F. Caselli, Diagonal invariants and the refined multimahonian distribution, J. Algebraic Combin., in press.

[11] C. Chevalley, Invariants of finite groups generated by reflections, Amer. J. Math. 77 (1955), 778-782

[12] A. Garsia and I. Gessel, Permutation statistics and partitions, Adv. Math. 31 (1979) 288-305.

[13] B. Gordon, Two theorems on multipartite partitions, J. London Math. Soc. 38 (1963) 459-464.

[14] W.Kraśkiewicz and J. Weyman, Algebra of coinvariants and the action of a Coxeter element, Bayreuth. Math. Schr. No. 63 (2001), 265-284.

[15] P. A. MacMahon, Combinatory analysis, vol. 1, Cambridge University Press, London, 1915.

[16] G. C. Shephard, Unitary groups generated by reflections, Canadian J. Math. 5 (1953), 364-383.

[17] G. C. Shephard and J. A. Todd, Finite unitary reflection groups, Canadian J. Math. 6 (1954), 274-304.

[18] L. Solomon, Partition identities and invariants of finite groups, J. Combin. Theory Ser. A 23 (1977), 148-175.

[19] R. P. Stanley, Invariants of finite groups and their applications to combinatorics, Bull. Amer. Math. Soc. 1 (1979), 475-511.

[20] R. P. Stanley, Enumerative combinatorics, vol. 2, Cambridge Studies in Advanced Mathematics 62, Cambridge University Press, Cambridge, 1999.

[21] D.W. Stanton and D.E. White, A Schensted algorithm for rim hook tableaux, J. Combin. Theory Ser. A 40 (1985), 211-247.

[22] J.R. Stembridge, On the eigenvalues of representations of reflection groups and wreath products. Pacific J. Math. 140 (1989), no. 2, 353-396.

E-mail address: caselli@dm. unibo.it 